\title{
Sub-wavelength diffraction-free imaging with low-loss metal-dielectric multilayers
}

\author{
Rafał Kotyński · Tomasz Stefaniuk • Anna Pastuszczak
}

Received: 30 March 2010 / Accepted: 13 December 2010 / Published online: 25 January 2011

(c) The Author(s) 2011. This article is published with open access at Springerlink.com

\begin{abstract}
We demonstrate numerically the diffraction-free propagation of sub-wavelength sized optical beams through simple elements built of metal-dielectric multilayers. The proposed metamaterial consists of silver and a high refractive index dielectric, and is designed using the effective medium theory as strongly anisotropic and impedance matched to air. Further it is characterised with the transfer matrix method, and investigated with FDTD. The diffraction-free behaviour is verified by the analysis of FWHM of PSF in the function of the number of periods. Small reflections, small attenuation, and reduced FabryPérot resonances make it a flexible diffraction-free material for arbitrarily shaped optical planar elements with sizes of the order of one wavelength.
\end{abstract}

\section{Introduction}

Since the seminal paper by Pendry [1], sub-wavelength imaging at visible wavelengths has been demonstrated in much thicker low-loss layered silver-dielectric periodic structures [2-7]. In fact, the effective medium theory (EMT) provides sufficient means to explain enhanced transmission through the metal-dielectric stacks. Sub-wavelength resolution results from the extreme anisotropy of the effective permittivity tensor $[2,8-10]$.

R. Kotyński ( $\varangle) \cdot$ T. Stefaniuk · A. Pastuszczak

Faculty of Physics, University of Warsaw, Poland Pasteura 7,

02-093 Warsaw, Poland

e-mail: rafal.kotynski@fuw.edu.pl

T. Stefaniuk

e-mail: tstefaniuk@igf.fuw.edu.pl

A. Pastuszczak

e-mail: apastuszczak@igf.fuw.edu.pl
Let us continue the introduction by linking the metaldielectric multilayers for sub-wavelength imaging with the concepts taken from Fourier Optics. We refer to the model of a linear shift invariant scalar system (LSI) [11, 12] for the description of optical multilayers in a situation when they act as imaging nano-elements for coherent monochromatic light. In-plane imaging through a layered structure consisting of uniform and isotropic materials represents a LSI, for either TE or TM polarisations. Linearity of the system is the consequence of linearity of materials and validity of the superposition principle for optical fields. Shift invariance results from the assumed infinite perpendicular size of the multilayer and the freedom in the choice of an optical axis.

A scalar description is valid for the TE and TM polarisations in 2D since all other field components may be derived from $E_{y}$ or $H_{y}$, respectively, where the co-ordinate system is oriented as in Fig. 1.

For the TM polarisation, the magnetic field $H_{y}(x, z)$ may be represented with its spatial spectrum $\hat{H}_{y}\left(k_{x}, z\right)$

$H_{y}(x, z)=\int_{-\infty}^{+\infty} \hat{H}_{y}\left(k_{x}, z\right) \exp \left(\imath k_{x} x\right) d k_{x}$

where, at least for lossless materials, the spatial spectrum is clearly separated into the propagating part $k_{x}^{2}<k_{0}^{2} \epsilon$ and evanescent part $k_{x}^{2}>k_{0}^{2} \epsilon$.

The transfer function $t\left(k_{x}\right)$ (TF) is the ratio of the output to incident fields spatial spectra and corresponds to the amplitude transmission coefficient of the multilayer

$\hat{H}_{y}\left(k_{x}, z=L\right)=t\left(k_{x}\right) \cdot \hat{H}_{y}^{\mathrm{Inc}}\left(k_{x}, z=0\right)$.

Due to reflections, the incident field $\hat{H}_{y}^{\mathrm{Inc}}\left(k_{x}, z=0\right)$ differs from the total field $\hat{H}_{y}\left(k_{x}, z=0\right)$.

The point spread function (PSF) is the inverse Fourier transform of the TF and has the interpretation of the re- 


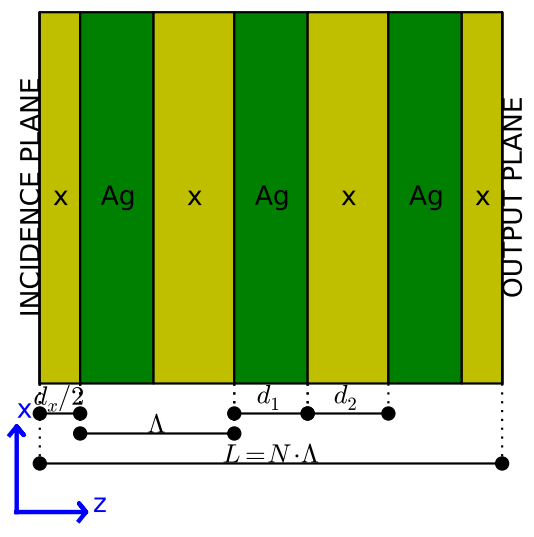

a
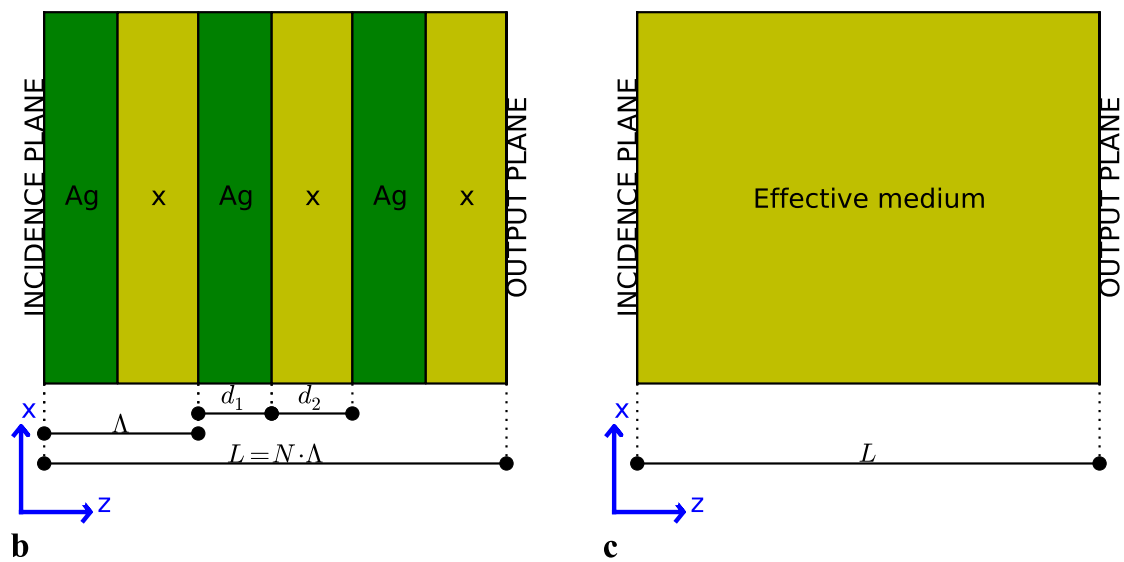

Fig. 1 Schematic of a periodic silver-dielectric multilayer with (a) symmetric composition, (b) non-symmetric composition, and (c) the equivalent effective medium slab

sponse of the system to a point signal $\delta(x)$. The response to an arbitrary input $H_{y}^{\text {Inc }}(x, z=0)$ can be further expressed as its convolution with the PSF

$H_{y}(x, z=L)=H_{y}^{\mathrm{Inc}}(x, z=0) * \operatorname{PSF}(x)$.

PSF of an imaging system usually provides clear information about the resolution, loss or enhancement of contrast, as well as the characteristics of image distortions. However, for sub-wavelength imaging, the PSF is not a straightforward measure of resolution and sometimes imaging of objects smaller than the FWHM of PSF is possible [13].

In this paper we demonstrate a diffraction-free material for sub-wavelength sized optical beams. We combine the following properties of the multilayer: PSF with subwavelength size and little dependence on the thickness of the structure, high transmission, low losses, and a limited dependence of the imaging properties on the size of external layers. Together, these properties allow one to use a multilayer as a flexible construction material for various optical imaging nano-devices.

\section{Imaging with sub-wavelength resolution in metal-dielectric multilayers}

The dispersion relation of a two-component infinite stack for the TM polarisation has the form [14]

$$
\begin{aligned}
\cos \left(K_{z} \Lambda\right)= & \cos \left(k_{1 z} f \Lambda\right) \cos \left(k_{2 z}(1-f) \Lambda\right) \\
& -\frac{\sin \left(k_{1 z} f \Lambda\right) \sin \left(k_{2 z}(1-f) \Lambda\right)}{2} \\
& \times\left(\frac{k_{1 z} \epsilon_{2}}{\epsilon_{1} k_{2 z}}+\frac{k_{2 z} \epsilon_{1}}{\epsilon_{2} k_{1 z}}\right),
\end{aligned}
$$

where $K_{z}$ is the Bloch wavenumber, $\Lambda=d_{1}+d_{2}$ is the period of the stack, $d_{i}$ and $\epsilon_{i}$ are the layer thickness and permittivity of material $i=1,2$, the filing fraction of material 1 is $f=d_{1} / \Lambda$ and the local dispersion relations are $k_{i z}^{2}+k_{x}^{2}=k_{0}^{2} \epsilon_{i}$. The wavevector $k_{x}$ is conserved at the layer boundaries and depends on the incidence conditions. The Bloch wavenumber $K_{z}$ is real for a Bloch mode in a lossless stack, however for evanescent waves in a finite stack or for lossy materials $K_{z}$ may be complex. In the first BZ, the real part of $K_{z}$ satisfies $\pi / \Lambda \leq r e\left(K_{z}\right) \leq \pi / \Lambda$. The group velocity as well as the imaginary part responsible for absorption do not depend on the choice of BZ.

When the layers are thin, $K_{z} \Lambda, k_{i z} \Lambda \ll 1$, the second order expansion of (4) over the arguments of trigonometric functions leads to the dispersion relation for an uniaxially anisotropic effective medium

$K_{z}^{2} / \epsilon_{x}+k_{x}^{2} / \epsilon_{z}=k_{0}^{2}$,

with $\epsilon_{x}=f \epsilon_{1}+(1-f) \epsilon_{2}$ and $\epsilon_{z}=1 /\left(f / \epsilon_{1}+(1-f) / \epsilon_{2}\right)$. This is the basis of the effective medium approximation thoroughly discussed by Wood et al. [2], also as the basis for applying the near-field approximation. The transmission coefficient of the Fabry-Pérot (FP) slab consisting of the homogenised effective medium is then given as [2]

$$
\begin{aligned}
t\left(k_{x}\right)= & \left(\cos \left(k_{z} L\right)-0.5 l\left(K_{z} \epsilon_{x} / k_{z} \epsilon\right.\right. \\
& \left.\left.+k_{z} \epsilon / K_{z} \epsilon_{x}\right) \sin \left(k_{z} L\right)\right)^{-1}
\end{aligned}
$$

where $k_{z}$ and $\epsilon$ refer to the external medium, and $L$ is the total thickness of the slab. At the same time $t\left(k_{x}\right)$ is the already mentioned coherent amplitude transfer function of the imaging system [12]. When $\left|\epsilon_{x} / \epsilon_{z}\right| \ll 1$ (which is satisfied for a lossless metal for $\epsilon_{1} / \epsilon_{2}=-d_{1} / d_{2}$ [9]), for certain slab thickness the resonant FP condition becomes independent on the angle of incidence. This happens when 


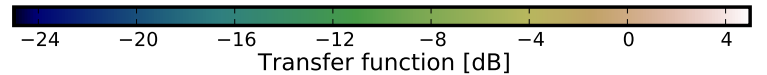

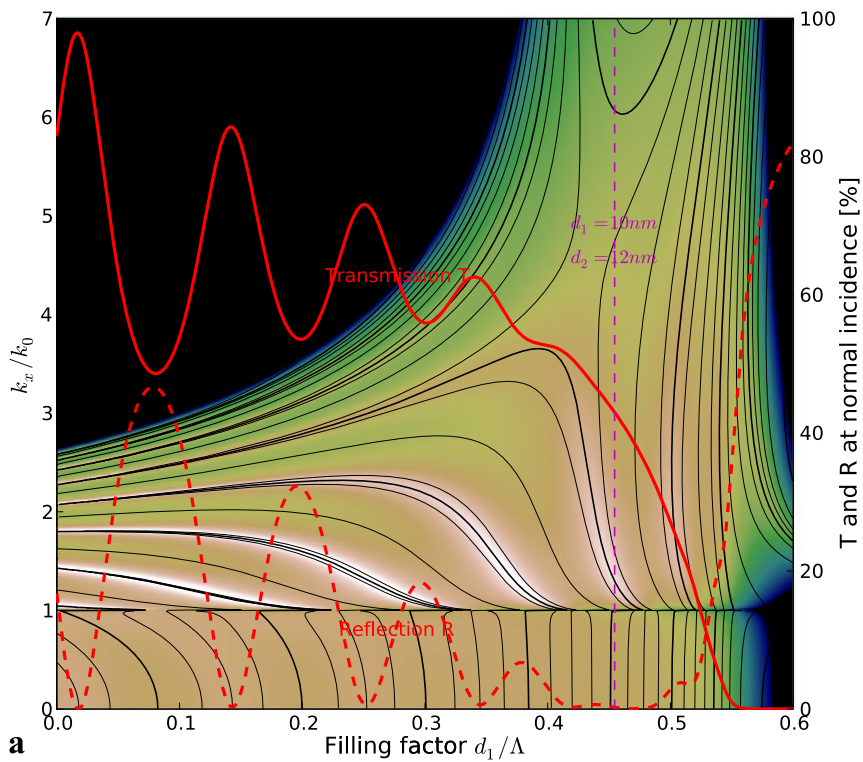

Fig. 2 (a) Intensity transmission and reflection coefficients $(T, R)$ of the symmetric multilayer as a function of the filling factor, plotted over the transfer function (in vertical cross sections of the colour map). Phase isolines are separated by $\pi / 4$. The pitch $\Lambda=d_{1}+d_{2}$ is fixed at $22 \mathrm{~nm}$, and the total thickness is $L=30 \Lambda$. (b) Characteristics of the

$L=\lambda m / \sqrt{\epsilon_{x}}, m \in N$. Then $t\left(k_{x}\right) \equiv \exp \left(-\imath K_{z} L\right)$ and the FP slab introduces the same phase shift for all harmonics of the spatial spectrum. Belov and Hao [9] proposed to combine this condition with impedance matching between the external medium and the effective FP slab $\epsilon=\epsilon_{x}=$ $f \epsilon_{1}+(1-f) \epsilon_{2}$ and referred to that regime as canalisation. However, Li et al. [6] questioned the importance of impedance matching in favour of the FP resonance condition. In fact, for a lossless metal and dielectric, the FP resonance is sufficient to entirely eliminate reflections resulting in perfect imaging $t\left(k_{x}\right) \equiv 1$ without impedance matching. In reality, losses make the condition $\epsilon=\epsilon_{x}$ only approximate and, at the same time, the finite value of $\Lambda$ limits the validity of homogenisation. Moreover, transmission through a finite slab strongly depends on the material and thickness of the external layers and appears to be the largest for a symmetrically designed slab (Fig. 1a) with half-width dielectric layers located at the boundaries [3].

\section{Numerical demonstration of diffraction-free propagation of sub-wavelength sized optical beams}

After recalling the theory of sub-wavelength imaging in thin metal-dielectric multilayers, we now focus on an imaging regime which may be called diffraction-free (due to the TF
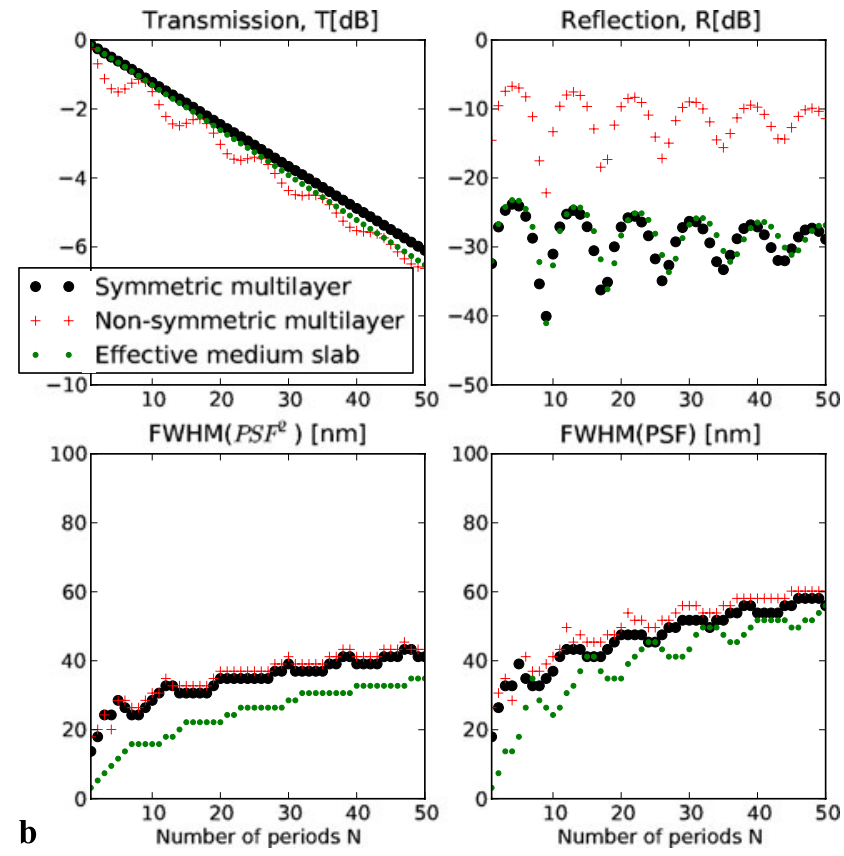

symmetric and non-symmetric multilayers, and of the equivalent slab made of an effective medium, in the function of the number of periods. Intensity transmission and reflection coefficients calculated at normal incidence (top); FWHM of PSF and $|\mathrm{PSF}|^{2}$ (bottom)

of the multilayer rather than the Bessel shape of the subwavelength sized beam as is reported in [15]). We note that the FP resonances are accompanied with a field pattern inside the slab similar to a standing wave $[2,7]$. Therefore we look for structures for which $t\left(k_{x}\right) \approx$ const nonetheless FP resonances are weak.

Let us now focus on an example of a metal-dielectric periodic multilayer consisting of silver and high refractive index dielectric, which enables us to demonstrate and explain the diffraction-free propagation of sub-wavelength sized optical beams. The structure operates at the wavelength of $\lambda=422 \mathrm{~nm}$, when the permittivity of silver is equal to $\epsilon_{1}=-5.637+0.214 l[16]$, and the permittivity of the dielectric such as $\mathrm{TiO}_{2}$ or $\mathrm{SrTiO}_{3}[11,17]$ is $\epsilon_{2}=(2.6)^{2}$. Layer thicknesses are assumed to be $d_{1}=10 \mathrm{~nm}, d_{2}=$ $12 \mathrm{~nm}$ with periodic symmetric or non-symmetric composition shown in Fig. 1a and b. The fraction $d_{1} / d_{2}$ may be justified with the use of EMT. The corresponding permittivity of the effective medium gives $\epsilon_{x} \approx 1.02+0.1 \mathrm{l}$ and $\epsilon_{z} \approx-158+191 l$, which ensure impedance matching with air $\epsilon=1 \approx \epsilon_{x}$ together with the condition for the extreme anisotropy $\left|\epsilon_{x} / \epsilon_{z}\right| \ll 1$. The imaginary part of the refractive index $n_{x}=\sqrt{\epsilon_{x}}$ is reduced by the factor of 50 compared to bulk silver, resulting in low-loss transmission. In Fig. 2a we show the transfer function calculated rigorously with TMM for a range of filling factors $0<d_{1} / \Lambda<0.6$, when the total thickness of the structure is fixed at $L=660 \mathrm{~nm}$. Indeed, 


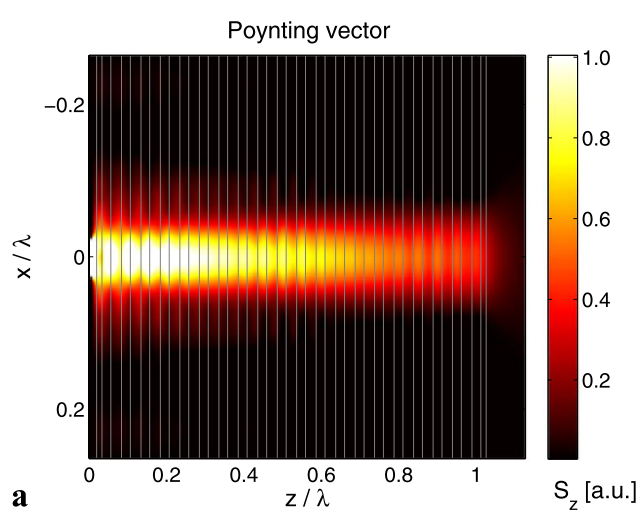

Fig. 3 Diffraction-free transmission of the sub-wavelength sized beam through the multilayer (FDTD simulations, with the incident CW beam limited by a sub-wavelength aperture in a perfect conductor). (a) Time-
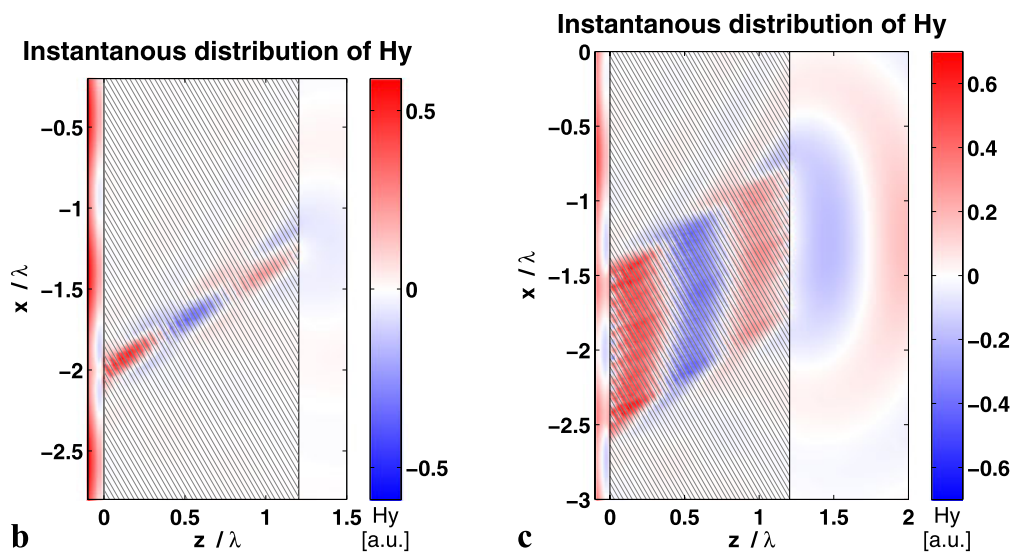

Fig. 4 Simple optical nano-elements internally made of the diffraction-free material. (a) Double slab. (b) Prism
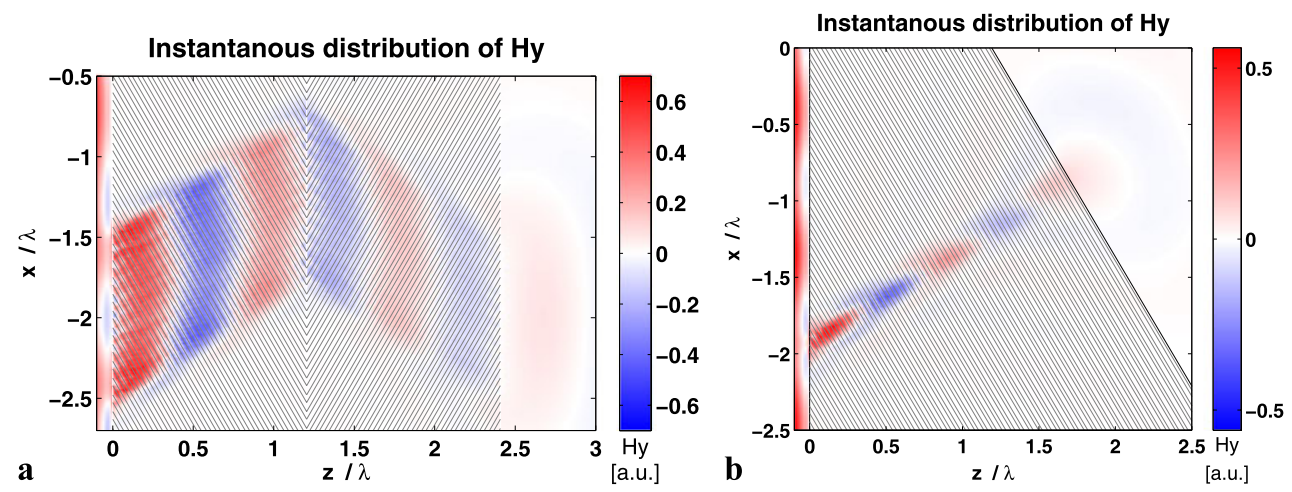

the impedance matching which occurs when $d_{1}=10 \mathrm{~nm}$, $d_{2}=12 \mathrm{~nm}$ results in reduced reflections, high transmission of both propagating and evanescent spatial harmonics, and a flat phase of the transfer function for a broad range of $k_{x} / k_{0}$. The size of the corresponding PSF is of the order of $\lambda / 10 \approx 40 \mathrm{~nm}$ ensuring the super-resolving properties of the device. Figure $2 b$ shows the FWHM of PSF alongside with the reflection and transmission coefficient in the function of number of periods, for the symmetric, nonsymmetric and effective-index composition of the structure. The major properties of the structure are the following: the size of PSF varies slowly with the size of the structure and is almost the same for the symmetric and non-symmetric composition, the FP resonances observed in transmission are weak (as opposed to those observed in reflections), the attenuation is uniform as the number of layers is increased. These properties ensure an almost diffraction-free propagation through the structure, with a similar attenuation and the size of PSF for symmetric and non-symmetric composition and for a broad range of structure sizes $L$. This behaviour is illustrated in Fig. 3a with an FDTD [18] simulation showing the uniform non-diverging distribution of the Poynting vector inside the structure for a beam size of the order of averaged Poynting vector $S_{z}$ in a rectangular slab. $(\mathbf{b}, \mathbf{c})$ Instantaneous magnetic field $H_{y}$ in a slab with layer boundaries oriented at $30^{\circ}$ toward the external boundaries for various aperture sizes 
PSF as a function of the number of periods. The material consists of silver and a dielectric with refractive index of $n=2.6$ and operates at $\lambda=422 \mathrm{~nm}$. It has the effective imaginary part of refractive index smaller than that of silver by a factor of 50 . The reflections are in between $-10 \mathrm{~dB}$ and $-30 \mathrm{~dB}$, and the FWHM of PSF is at the order of $40 \mathrm{~nm}$. Small reflections, small attenuation, and reduced Fabry-Pérot resonances make it a flexible metamaterial for arbitrarily shaped optical planar devices with sizes of the order of one wavelength, such as the elements of optical interconnects or cloaks.

Acknowledgement We acknowledge support from the Polish Ministry of Science and Higher Education research project N N202 033237, the National Centre for Research and Development research project N R15 0018 06, and the framework of European Cooperation in Science and Technology-COST action MP0702.

Open Access This article is distributed under the terms of the Creative Commons Attribution Noncommercial License which permits any noncommercial use, distribution, and reproduction in any medium, provided the original author(s) and source are credited.

\section{References}

1. J.B. Pendry, Negative refraction makes a perfect lens. Phys. Rev. Lett. 85(18), 3966-3969 (2000)

2. B. Wood, J.B. Pendry, D.P. Tsai, Directed subwavelength imaging using a layered metal-dielectric system. Phys. Rev. B 74, 115116 (2006)

3. M. Scalora, G. D'Aguanno, N. Mattiucci, M.J. Bloemer, D. Ceglia, M. Centini, A. Mandatori, C. Sibilia, N. Akozbek, M.G. Cappeddu, M. Fowler, J. Haus, Negative refraction and sub-wavelength focusing in the visible range using transparent metallo-dielectric stacks. Opt. Express 15, 508-523 (2007)

4. D. de Ceglia, M.A. Vincenti, M.G. Cappeddu, M. Centini, N. Akozbek, A. D’Orazio, J.W. Haus, M.J. Bloemer, M. Scalora,
Tailoring metallodielectric structures for superresolution and superguiding applications in the visible and near-IR ranges. Phys. Rev. A 77, 033848 (2008)

5. N. Mattiucci, G. D’Aguanno, M. Scalora, M.J. Bloemer, C. Sibilia, Transmission function properties for multi-layered structures: application to super-resolution. Opt. Express 17, 17517-17529 (2009)

6. X. Li, S. He, Y. Jin, Subwavelength focusing with a multilayered Fabry-Pérot structure at optical frequencies. Phys. Rev. B 75(4), 045103 (2007)

7. R. Kotynski, T. Stefaniuk, Comparison of imaging with subwavelength resolution in the canalization and resonant tunnelling regimes. J. Opt. A, Pure Appl. Opt. 11, 015001 (2009)

8. P.A. Belov, C. Simovski, P. Ikonen, Canalization of subwavelength images by electromagnetic crystals. Phys. Rev. B 71(19), 193105 (2005)

9. P.A. Belov, Y. Hao, Subwavelength imaging at optical frequencies using a transmission device formed by a periodic layered metaldielectric structure operating in the canalization regime. Phys. Rev. B 73, 113110 (2006)

10. Q.M. Quan, S.L. Zhu, R.P. Wang, Refraction in the fixed direction at the surface of dielectric/silver superlattice. Phys. Lett. A 359 , 547-549 (2006)

11. B. Saleh, M. Teich, Fundamentals of Photonics, 2nd edn. (Wiley, New York, 2007)

12. J.W. Goodman, Introduction to Fourier Optics, 3rd edn. (Roberts, Greenwood Village, 2005)

13. R. Kotynski, T. Stefaniuk, Multiscale analysis of subwavelength imaging with metal-dielectric multilayers. Opt. Lett. 35, 11331135 (2010)

14. L. Wu, S. He, L.F. Shen, Band structure for a one-dimensional photonic crystal containing left-handed materials. Phys. Rev. B 67, 235103 (2003)

15. J.J. Miret, C.J. Zapata-Rodriguez, Surface-assisted ultralocalization in nondiffracting beams (2010). arXiv:1001.3204v1

16. P. Johnson, R. Christy, Optical constants of the noble metals. Phys. Rev. B 6, 4370-4379 (1972)

17. E. Palik (ed.), Handbook of Optical Constants of Solids (Academic Press, San Diego, 1998)

18. A. Farjadpour, D. Roundy, A. Rodriguez, M. Ibanescu, P. Bermel, J.D. Joannopoulos, S.G. Johnson, G. Burr, Improving accuracy by subpixel smoothing in FDTD. Opt. Lett. 31, 2972-2974 (2006) 